\section{§15. Particle Reflections of Low Energy Light Ions from a Vanadium Alloy $(\mathrm{V}-4 \mathrm{Cr}-4 \mathrm{Ti})$}

Yamaoka, H. (RIKEN),

Tanaka, N., Takeuchi, S., Sugawara, H.,

Okamoto, A., Sasao, M. (Tohoku Univ.),

Matsumoto, Y. (Tokushima Bunri Univ.),

Nishiura, M.,

Wada, M. (Doshisha Univ.)

We have been developing an experimental system to study interactions of beams with solid surfaces. The system has successfully produced ion spectra reflected from Mo, carbon coated Mo, and $\mathrm{W}$ targets. ${ }^{1-3)}$ Here we report the first results of the study on particle reflections from the NIFS-HEATs vanadium alloy, bombarded by low energy (1-2 keV) hydrogen ions. ${ }^{4)}$ Figure 1 shows the energy and angular resolved intensity contour map of $\mathrm{H}^{+}$and $\mathrm{H}^{-}$ions at the incident angle of $20^{\circ}$ at room temperature. The vanadium alloy surface shows a characteristic reflection angle dependence of the reflected ion energy with the time-dependent change at a constant temperature. The energy spectra of the reflected ions from the vanadium target depend upon the reflection angle more strongly than other targets. Figure 2 shows angular distribution of the reflected positive and negative ion intensities, where $\alpha$ and $\beta$ are incident and reflection angles, respectively. Mirror reflections $(\alpha=\beta)$ are shown by the solid lines. For $\mathrm{H}^{+}$beam injection the deviation from the mirror reflection seems to be large at lower incident angle less than $\alpha=30^{\circ}$, even if we take the error of the target angle into account. Some inter-metallic compounds including vanadium are known as a material to absorb hydrogen and oxygen. We expected the characteristics of the ion reflection would differ from those of other materials depending on the sample temperature and the time to bake out the surface. In the experiment once the target was exposed in the air. After the evacuation, the sample was heated up. Before any heating, the intensity of the reflected $\mathrm{H}^{+}$beam was relatively large, probably due to the hydrogen or water adsorption on the sample surface. After heating the sample at $240-260^{\circ} \mathrm{C}$ for 30 min, the reflected $\mathrm{H}^{+}$intensity decreased drastically and then took the minimum values. It recovered slightly after $270 \mathrm{~min}$. As time passes, the angular dependence of the reflected $\mathrm{H}^{+}$ intensity became closer to mirror reflection.

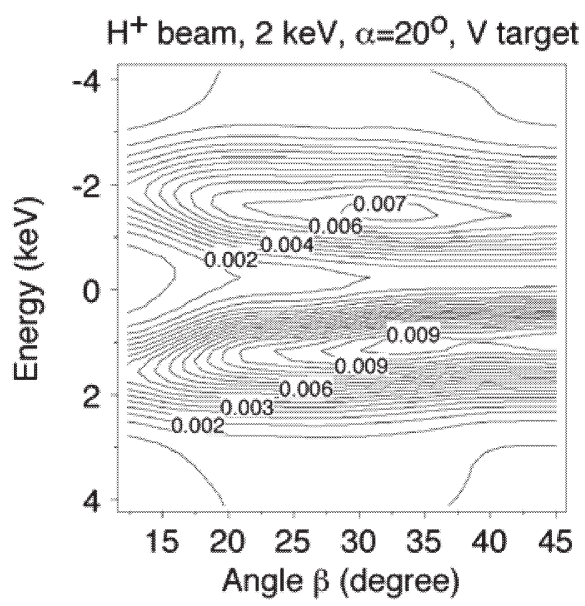

Fig. 1 Contour intensity plot of intensity profiles of positive and negative ions reflected from vanadium alloy surface.

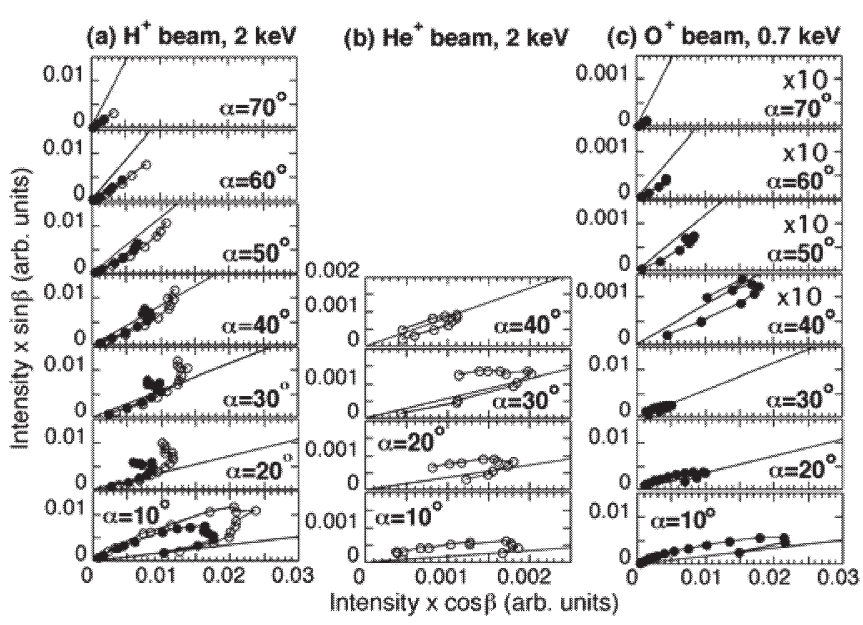

Fig. 2 Angular distribution of the intensity of reflected positive and negative ions for (a) $\mathrm{H}^{+}$beam of $2.0 \mathrm{keV}$, (b) $\mathrm{He}^{+}$ beam of $2.0 \mathrm{keV}$ and (c) $\mathrm{O}^{+}$beam of $0.7 \mathrm{keV}$. Open and closed circles correspond positive and negative ions, respectively.

\section{References}

1) M. Wada et al., Rev. Sci. Instrum. 73 (2002) 955.

2) H. Yamaoka et al., J. Nucl. Mater. $337-339$ (2005) 942.

3) H. Yamaoka et al., Rev. Sci. Instrum. 77 (2006) 03C301.

4) H. Yamaoka et al., J. Nucl Mater. 363-365 (2007) 1304. 